\title{
ON REMOTE POINTS IN $v X-X$
}

\author{
TOSHIJI TERADA
}

\begin{abstract}
Under a certain set-theoretic assumption, a question of E. K. van Douwen is solved. More precisely, if the cellularity $c(X)$ of a space $X$ is nonmeasurable, then $v X-X$ contains no remote point of $X$.
\end{abstract}

All spaces considered here are Tychonoff. For a space $X, \beta X$ is the Stone-Cech compactification and $v X$ is the Hewitt realcompactification of $X$. A point $p \in \beta X-X$ is said to be remote if $p \notin \mathrm{Cl}_{\beta X} D$ for every nowhere dense subset $D$ of $X$. In [1], Eric K. van Douwen discussed fully the theory of remote points. He raised the following question: Can $v X-X$ contain a remote point of $X$ ?

In this note we shall show that under a certain set-theoretic assumption $v X-X$ cannot contain a remote point of $X$.

A cardinal $m$ is called measurable if a set $X$ of cardinality $m$ admits a $\{0,1\}$-valued measure $\mu$ such that $\nu(X)=1$, and $\mu(\{x\})=0$ for every $x \in X$. The proposition that no measurable cardinal exists is known to be consistent with ZFC (see [2]). Let us recall a cardinal function given in [3]. The cellularity of a space $X$ is $c(X)=\sup \{|\mathcal{Q}|: \mathcal{Q}$ is a family of pairwise disjoint nonempty open subsets of $X$ \}.

THEOREM. For a space $X$, if $c(X)$ is nonmeasurable, then $v X-X$ contains no remote point of $X$.

Proof. Let $p$ be a point of $v X-X$. Let $\mathcal{U}$ be a maximal collection of pairwise disjoint nonempty open subsets of $X$ such that $p \notin \mathrm{Cl}_{\beta X} U$ for each $U \in \mathcal{Q}$. Then $|\mathcal{Q}|$ is nonmeasurable since $|\vartheta| \leqslant c(X)$. Let $D=X-\cup \mathscr{U}$, where $\cup \mathscr{U}=\cup\{U: U \in \mathcal{U}\}$. Then $D$ is a nowhere dense subset of $X$. Hence to see that $p$ is not a remote point of $X$ it suffices to show that $p \in \mathrm{Cl}_{\beta X} D$. Assume that $p \notin \mathrm{Cl}_{\beta X} D$. Let

$$
\mathfrak{F}=\left\{\mathfrak{V}: \mathfrak{V} \subset \mathcal{Q}, p \in \operatorname{Ex}_{X}(\cup \mathfrak{V})\right\}
$$

where $\operatorname{Ex}_{X} U=\beta X-\mathrm{Cl}_{\beta X}(X-U)$ for every open subset $U$ of $X$. We shall show that $\mathfrak{F}$ is an ultrafilter on $\mathscr{U}$ with the countable intersection property. Since

$$
\operatorname{Ex}_{X}\left(\cap\left\{U_{i}: i=0,1, \ldots, n\right\}\right)=\cap\left\{\operatorname{Ex}_{X} U_{i}: i=0,1, \ldots, n\right\}
$$

for each finite collection $\left\{U_{i}: i=0,1, \ldots, n\right\}$ of open subsets of $X$ (see [1]),

Received by the editors November 1, 1978.

AMS (MOS) subject classifications (1970). Primary 54D35.

Key words and phrases. Remote point, realcompactification, measurable cardinal. 
$\mathfrak{F}$ has the finite intersection property. Let $\mathfrak{V}$ be a subcollection of $\mathscr{U}$. Since

$$
\begin{aligned}
& \beta X=\mathrm{Cl}_{\beta X}(\cup \mathfrak{V}) \cup \mathrm{Cl}_{\beta X}(\cup(\mathscr{Q}-\mathfrak{V})) \\
& =\left(\operatorname{Bd}_{\beta X}\left(\operatorname{Ex}_{X}(\cup \mathcal{V})\right) \cup \operatorname{Ex}_{X}(\cup \mathcal{V})\right) \\
& \cup\left(\operatorname{Bd}_{\beta X}\left(\operatorname{Ex}_{X}(\cup(\mathcal{U}-\mathfrak{V}))\right) \cup \operatorname{Ex}_{X}(\cup(\mathcal{U}-\mathfrak{V}))\right) \\
& =\left(\mathrm{Cl}_{\beta X}\left(\mathrm{Bd}_{X}(\cup \mathfrak{V})\right) \cup \operatorname{Ex}_{X}(\cup \mathfrak{V})\right) \\
& \cup\left(\mathrm{Cl}_{\beta X}\left(\mathrm{Bd}_{X}(\cup(\mathscr{Q}-\mathfrak{V}))\right) \cup \operatorname{Ex}_{X}(\cup(\mathscr{Q}-\mathfrak{V}))\right) \\
& =\mathrm{Cl}_{\beta X} D \cup \operatorname{Ex}_{X}(\cup \mathcal{V}) \cup \operatorname{Ex}_{X}(\cup(\mathscr{Q}-\mathscr{V}))
\end{aligned}
$$

and $p \notin \mathrm{Cl}_{\beta X} D$, it is obvious that $p \in \operatorname{Ex}_{X}(\cup \mathfrak{V})$ or $p \in \operatorname{Ex}_{X}(\cup(\mathcal{Q}-\mathscr{V})$ ). This implies that $\mathfrak{F}$ is an ultrafilter on $\mathcal{Q}$. Let us show that $\mathfrak{F}$ has the countable intersection property. Assume that there is a sequence $\left\{\mathscr{V}_{i}: i<\omega\right\}$ of elements of $\mathfrak{F}$ such that

$$
\mathfrak{V}=\cap\left\{\mathfrak{V}_{i}: i<\omega\right\} \notin \mathfrak{F}
$$

Then $p \notin \mathrm{Cl}_{\beta X}(\cup \mathcal{W})$ since

$$
\mathrm{Cl}_{\beta X}(\cup \mathcal{V})=\operatorname{Ex}_{X}(\cup \mathcal{V}) \cup \mathrm{Bd}_{\beta X}\left(\operatorname{Ex}_{X}(\cup \mathcal{V})\right)
$$

$p \notin \operatorname{Ex}_{X}(\cup \mathcal{V})$ and $p \notin \mathrm{Bd}_{\beta X}\left(\operatorname{Ex}_{X}(\cup \mathcal{V})\right)=\mathrm{Cl}_{\beta X}\left(\mathrm{Bd}_{X}(\cup \mathcal{V})\right) \subset \mathrm{Cl}_{\beta X} D$ Hence there is a zero-set $Z_{\omega}$ of $\beta X$ such that $p \in Z_{\omega}$ and $Z_{\omega} \cap \mathrm{Cl}_{\beta X}(\cup \mathcal{V})=$ $\varnothing$. On the other hand, for each $i<\omega$ there is a zero-set $Z_{i}$ of $\beta X$ such that $p \in Z_{i}$ and $Z_{i} \subset \operatorname{Ex}_{X}\left(\cup \mathfrak{V}_{i}\right)$. Now, let $Z=\cap\left\{Z_{i}: i \leqslant \omega\right\}$. Then $Z$ is a zero-set of $\beta X$ which contains $p$. Since

$$
\begin{gathered}
\cup \mathcal{V}=\cup\left(\cap\left\{\mathscr{V}_{i}: i<\omega\right\}\right)=\cap\left\{\cup \mathscr{V}_{i}: i<\omega\right\} \\
Z \cap X \subset\left(\cap\left\{\cup \mathcal{V}_{i}: i<\omega\right\}\right) \cap\left(X-\mathrm{Cl}_{X}(\cup \mathcal{V})\right)=\varnothing
\end{gathered}
$$

But this is a contradiction since $p \in v X$. Hence $\mathfrak{F}$ has the countable intersection property. But, since $\{U\} \notin \mathfrak{F}$ for each $U \in \mathcal{U}$, this contradicts the fact that $|\mathcal{Q}|$ is nonmeasurable (see [2]).

Corollary 1. Assume that every cardinal is nonmeasurable. Then $v X-X$ contains no remote point of $X$ for any space $X$.

Corollary 2. Assume that every cardinal is nonmeasurable. If $X$ has a remote point, then $X$ is not pseudocompact.

Corollary 2 shows that nonpseudocompactness is essential to have a remote point.

REMARK. The converse of the above Theorem is not true (i.e. the nonmeasurability of $c(X)$ need not be implied by the fact that $v X-X$ contains no remote point of $X$ ). However the nonmeasurability of $c(X)$ cannot be dropped in the Theorem. In fact, let $M$ be a discrete space of measurable 
cardinality. Then $v M-M$ is nonempty, and every point of $v M-M$ is a remote point of $M$.

\section{REFERENCES}

1. E. K. van Douwen, Remote points, Dissertationes Math. (to appear).

2. L. Gillman and M. Jerison, Rings of continuous functions, Van Nostrand, Princeton, N. J., 1960.

3. I. Juhász, Cardinal functions in topology, Math. Centre Tract No. 34, Mathematisch Centrum, Amsterdam, 1971.

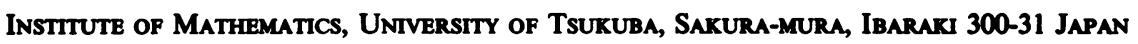

